\title{
Strong CD8+ lymphocyte infiltration in combination with expression of HLA class I is associated with better tumor control in breast cancer patients treated with neoadjuvant chemotherapy
}

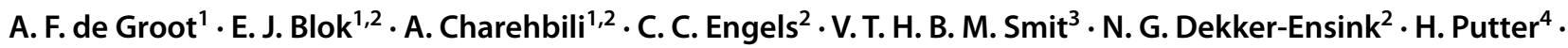 \\ E. Meershoek-Klein Kranenbarg ${ }^{2}$ - C. J. H. van de Velde ${ }^{2}$. G. J. Liefers ${ }^{2}$ • J. W. R. Nortier ${ }^{1}$ • P. J. K. Kuppen ${ }^{2}$. \\ S. H. van der Burg' ${ }^{1}$ J. R. Kroep ${ }^{1}$
}

Received: 6 December 2018 / Accepted: 5 March 2019 / Published online: 13 March 2019

(c) The Author(s) 2019

\begin{abstract}
Purpose Tumor-infiltrating lymphocytes (TILs) are associated with pathological complete response (pCR) and survival after neoadjuvant chemotherapy (NAC) in patients with early breast cancer. We investigated the prognostic and predictive role of TILs, macrophages, and HLA class 1 expression after NAC with or without the potentially immune modulating compound zoledronic acid (ZA).

Methods Baseline tumor biopsies from 196 patients in the NEOZOTAC trial were analyzed for CD8 (cytotoxic T-cells), FoxP3 (regulatory T-cells), CD68 (macrophages), and HLA class I (HCA2/HC10) expression by immunohistochemistry and subsequently related to pCR and disease-free survival (DFS).

Results A strong intratumoral CD8+ infiltration or expression of HLA class 1 by cancer cells was associated with a higher pCR rate $(p<0.05)$. Clinical benefit of high CD8+ T-cell infiltration was found when cancer cells expressed HLA class 1 (pCR: $21.8 \%$ vs. $6.7 \%, p=0.04$ ) but not when HLA class 1 expression was lost or downregulated (pCR: $5.9 \%$ vs. $0 \%$, $p=0.38$ ). Interaction analyses revealed survival benefit between HLA class 1 expression and strong CD8+ T-cell infiltration, whereas in the absence or downregulation of HLA class 1 expression, high levels of CD8+ T-cells were associated with survival disadvantage ( $\mathrm{p}$ for interaction 0.01 ; hazard ratio $0.41,95 \%$ CI $0.15-1.10, p=0.08$ and hazard ratio $7.67,95 \%$ CI $0.88-66.4, p=0.07$, respectively). Baseline immune markers were not related to ZA treatment.

Conclusions Strong baseline tumor infiltration with CD8+ T-cells in the presence of tumoral HLA class 1 expression in patients with HER2-negative breast cancer is related to a higher pCR rate and a better DFS after NAC.
\end{abstract}

Keywords Neoadjuvant chemotherapy $\cdot$ Breast cancer $\cdot$ Zoledronic acid $\cdot$ Tumor-infiltrating lymphocytes $\cdot$ HLA class 1 . Pathological complete response

Electronic supplementary material The online version of this article (https://doi.org/10.1007/s10549-019-05195-y) contains supplementary material, which is available to authorized users.

\section{J. R. Kroep}

j.r.kroep@lumc.nl

1 Department of Medical Oncology, Leiden University Medical Center, Albinusdreef 2, 2333 ZA Leiden, The Netherlands

2 Department of Surgery, Leiden University Medical Center, Albinusdreef 2, 2333 ZA Leiden, The Netherlands

3 Department of Pathology, Leiden University Medical Center, Albinusdreef 2, 2333 ZA Leiden, The Netherlands

4 Department of Statistics, Leiden University Medical Center, Albinusdreef 2, 2333 ZA Leiden, The Netherlands

$\begin{array}{ll}\text { Abbreviations } \\ \text { CTLs } & \text { Cytotoxic T-cells } \\ \text { DAB } & 3,3^{\prime} \text {-Diaminobenzidine } \\ \text { DFS } & \text { Disease-free survival } \\ \text { ER } & \text { Estrogen receptor } \\ \text { FFPE } & \text { Formalin-fixed paraffin-embedded } \\ \text { H\&E } & \text { Hematoxylin and eosin stained } \\ \text { HR } & \text { Hormone receptor } \\ \text { NAC } & \text { Neoadjuvant chemotherapy } \\ \text { pCR } & \text { Pathological complete response } \\ \text { PR } & \text { Progesterone receptor } \\ \text { TAMs } & \text { Tumor-associated macrophages } \\ \text { TILs } & \text { Tumor-infiltrating lymphocytes } \\ \text { TNBC } & \text { Triple-negative breast cancer }\end{array}$


Tregs Regulatory T-cells

ZA Zoledronic acid

\section{Background}

For patients with locally advanced breast cancers, neoadjuvant chemotherapy (NAC) is an increasingly used treatment modality. The main goal of neoadjuvant therapy is to enable breast-conserving therapy and spare patients from an axillary lymph node dissection. Additionally, neoadjuvant studies make use of the possible accelerated drug selection for evaluation in adjuvant phase III early breast cancer trials [1]. Studies have shown that the occurrence of pathological complete response (pCR) after neoadjuvant therapy is associated with improved survival, especially in non-luminal type tumors [2-4].

Evaluation of tumor-infiltrating lymphocytes (TILs) on both hematoxylin and eosin (H\&E) stained and immunohistochemical stained tumor sections has demonstrated promising value as a predictor of $\mathrm{pCR}$ and survival after neoadjuvant therapy, especially in patients with estrogen receptor (ER)-negative and HER2-positive disease [5-7], and efforts are being made for harmonization of this parameter by a dedicated international TIL-working group [8-10]. However, the composition of TILs is typically heterogeneous and includes cytotoxic T-cells (CTLs), targeting tumor cells, as well as immunosuppressing regulatory T-cells (Tregs) with potentially different implications for the chances of achieving $\mathrm{pCR}$ and improving survival $[11,12]$. A recent metaanalysis by Wang et al. confirmed the findings of several studies by showing a positive association between higher levels of CD8+ CTL infiltrate and pCR [5]. Data on the relation between CD8+ CTL infiltrate and survival after NAC are less extensive, but some studies suggest high CD8+ CTL infiltrate to be prognostic for an improved survival [13-15]. For FoxP3+ Treg infiltrate, meta-analyses by Wang et al. and Mao et al. reported contradictory results regarding an association with pCR $[5,16]$ and also studies on an association with survival reported discordant results [15, 17-19]. Tumor-associated macrophages (TAMs) may also play a role in the tumor microenvironment [20], but studies on (CD68+) TAM infiltrate as a biomarker in the neoadjuvant setting are scarce $[21,22]$. In general, it is thought that patients with high numbers of TAM infiltrate are likely to have a worse survival rate [20]. Importantly, tumor cells can acquire features to escape from immune recognition by downregulation of HLA class 1 expression [23]. Previously, de Kruijf et al. observed that loss and downregulation of HLA class 1 resulted in worse outcome in terms of breast cancer relapse [12]. However, only few studies have investigated the role of HLA class 1 expression and results are discordant [12, 24-26].
In this study, we aimed to investigate the role of CTLs, Tregs, and TAMs in the tumor microenvironment in the context of HLA class 1 expression in a study cohort consisting of patients treated with NAC with or without zoledronic acid (ZA) in a randomized phase III trial [27]. As ZA is suggested to have immunomodulatory features [28], our secondary aim was to investigate whether there were differential treatment benefits of ZA between biomarker-based subgroups with regard to pCR and disease-free survival (DFS).

\section{Methods}

\section{Patients and tumor material}

Eligibility criteria for the NEOZOTAC study have been described previously [27]. In brief, patients with stage II/III, HER2-negative breast cancer were randomized to 6 cycles of NAC with or without ZA. Medical-ethical approval was obtained and the study was conducted in accordance with the Declaration of Helsinki. All patients signed informed consent before randomization. Formalin-fixed paraffin-embedded (FFPE) samples were collected from pre-NAC biopsies. All samples were handled in a coded fashion, according to national ethical guidelines ("Code for Proper Secondary Use of Human Tissue," Dutch Federation of Medical Scientific Societies). Central data collection was done at the Datacenter of the Department of Surgery of the LUMC. Biomarkers were reported according to the REMARK criteria [29].

\section{Antibodies}

Mouse monoclonal antibodies against CD8 (ab17147 clone 144B; Abcam (1:25) and clone 4B11; Monosan (1:25)), FoxP3 (ab20034 clone 236A/E7; Abcam (1:200)), and CD68 (M0814 clone KP1; Dako (1:15.000)) were used for immunohistochemical identification of CTLs, Tregs, and macrophages, respectively. For HLA class 1, mouse monoclonal antibodies HCA2 (1:200) and HC10 (1:400) were used. The HCA2 antibody binds to all HLA-A chains (except HLA-A24) and some of the HLA-B/HLA-C/HLA-E/HLA-F/ HLA-G chains [30, 31]. The HC10 antibody binds to HLAB, HLA-C, and some HLA-A chains (HLA-A3/HLA-A10/ HLA-A28/HLA-A29/HLA-A30/HLA-A31/HLA-A32/HLAA33) [32-34]. Antibodies were kindly provided by Prof. Dr. J. Neefjes.

\section{Immunohistochemistry}

Immunohistochemical stains were performed on 4- $\mu \mathrm{m} \mathrm{sec-}$ tions from FFPE blocks with core needle tumor biopsies. 
Tissue sections were deparaffinized and rehydrated. Slides were peroxidase blocked for $20 \mathrm{~min}$ in $\mathrm{H}_{2} \mathrm{O}_{2}$ solution $(0.03 \%)$. Antigen retrieval was performed in PT link (Dako) (low $\mathrm{pH}$ for CD8/CD68, high $\mathrm{pH}$ for FoxP3) for $10 \mathrm{~min}$. After incubation with secondary antibody envision antimouse (Dako Cytomation K4001), sections were visualized using 3,3'-diaminobenzidine (DAB+; K3468, Dako). Sections were counterstained with hematoxylin, dehydrated and mounted in Pertex. For HCA2/HC10, stains were performed using the DAKO autostainer. Human tonsil or placenta tissue served as a positive control for each staining. Slides that underwent the complete immunohistochemical staining procedure without primary antibody served as negative controls.

\section{Evaluation of immunostaining}

Microscopic analyses of HCA2, HC10, and FoxP3+ lymphocyte counts were performed blinded by two independent observers (A.C. $100 \%$ of cohort, C.E. $30 \%$ of cohort). Analyses of CD8+ lymphocyte and CD68+ macrophage counts were performed using the Vectra 3.0, an automated quantitative pathology imaging system with InForm software (PerkinElmer, Waltham MA).

The percentage of tumor cells showing membranous staining for HCA2/HC10 was estimated. Expression status of HLA class 1 was defined according to the International HLA and Immunogenetics Workshop: downregulation of tumor cell HLA class 1 expression was defined as less than $5 \%$ of tumor cells staining positive for either HCA2 or $\mathrm{HC} 10$ and loss of tumor cell HLA class 1 expression as less than $5 \%$ of tumor cells staining positive for both HCA2 and $\mathrm{HC} 10$ [35]. A dichotomous variable for loss + downregulation $(\mathrm{HCA} 2$ and/or $\mathrm{HC} 10<5 \%)$ vs. expression (HCA2 and $\mathrm{HC} 10 \geq 5 \%$ ) was calculated.

Quantification of the number of tumoral FoxP3+ lymphocytes was done by assessment of three random tumorcontaining fields at a $\times 20$ magnification. For quantification, a reticle grid (incorporated in the microscope ocular) with 1 square representing $0.36 \mathrm{~mm}^{2}$ was used for manual counting of positive lymphocytes. The lymphocyte counts in 3 squares were averaged for the lymphocyte score. A variable for FoxP3 status (high/low) was calculated using the median as cut-off value.

In brief, for quantification of the number of CD8+ and CD68+ cells, slides were scanned for image acquisition $(\times 4$ magnification). Subsequently, ten multispectral images per slide on average were selected manually $(\times 20$ magnification, one image representing roughly $0.33 \mathrm{~mm}^{2}$ ). With InForm software, the computer was trained to segment stroma, tumor, and other tissue (e.g., adipose tissue/empty spaces) and to distinguish between DAB-positive CD8/CD68 cells and DAB-negative cells with a learn-by-example interface.
The resulting algorithm was tested for accuracy in several images before being used for all images, where after the number of CD8+ or CD68+ cells per $\mathrm{mm}^{2}$ tumor could be calculated. A variable for CD8 and CD68 status (high/low) was calculated using the median as cut-off value. The creation of the algorithms was done by two researchers (AG/EB) under supervision of a pathologist (VS).

\section{Statistical analyses}

Statistical analyses were performed using SPSS (v.23.0 for Windows, IBM SPSS statistics). Patients of whom tumor material was lost during or appeared non-evaluable after staining were excluded from analyses. Cohen's kappa coefficient was used to assess inter-observer agreement in manual quantification of immunohistochemical stains (HCA2/HC10/ FoxP3). Clinical endpoints examined were pCR, defined as absence of residual tumor in the breast and lymph nodes, and DFS, defined as time from randomization to first relapse, second primary invasive breast cancer or death (any cause). Associations between markers and pCR were tested using Chi-square and Fisher's exact tests. Survival analyses were performed using Kaplan-Meier curves and Cox regression analyses. Median follow-up time (4.6 years) was determined using the reverse Kaplan-Meier method. For multivariable analyses, logistic regression was used.

\section{Results}

Of 196 out of 250 patients, core biopsy material before treatment was available for evaluation. Patient and tumor characteristics are described in Table 1. An overview of these characteristics for each separate staining can be found in Online Resource 1. Substantial agreement (kappa $\geq 0.6$ ) was observed for quantification of the immunohistochemical stains for FoxP3, HCA2, and HC10. Representative images of immunohistochemical stains for HLA class 1, CD8, FoxP3, and CD68 are shown in Fig. 1.

\section{Pre-treatment immune markers and associations with pCR}

Successful staining and scoring of CD8+ T-cells, FoxP3+ T-cells and CD68+ macrophages was obtained in $82 \%$ $(n=160), 80 \%(n=156)$, and $83 \%(n=163)$ of biopsies, respectively. An overview of general statistics for each immune marker for the whole group and stratified on allocated treatment can be found in Online Resource 2. When the group of patients was divided based on the median cell count per $\mathrm{mm}^{2}$ in the tumor area, higher pCR rates were observed in patients with high (above median) CD8+ infiltrate compared to low (below median) CD8+ infiltrate (pCR: 
Table 1 Patient and tumor characteristics of the study cohort

\begin{tabular}{|c|c|c|}
\hline \multirow[t]{2}{*}{ Parameter } & \multicolumn{2}{|c|}{$\begin{array}{l}\text { Study cohort (total } \\
N=196 \text { ) }\end{array}$} \\
\hline & $N$ & $\%$ \\
\hline \multicolumn{3}{|l|}{ Age } \\
\hline$<55$ years & 144 & 73.5 \\
\hline $55-64$ years & 44 & 22.4 \\
\hline$>64$ years & 8 & 4.1 \\
\hline \multicolumn{3}{|l|}{ HR status } \\
\hline $\mathrm{HR}-(\mathrm{TNBC})$ & 32 & 16.3 \\
\hline $\mathrm{HR}+$ & 164 & 83.7 \\
\hline \multicolumn{3}{|l|}{ Clinical tumor stage } \\
\hline $\mathrm{cT} 1$ & 1 & 0.5 \\
\hline cT2 & 111 & 56.6 \\
\hline cT3/cT4 & 84 & 42.9 \\
\hline \multicolumn{3}{|c|}{ Clinical lymph node stage } \\
\hline $\mathrm{cN}-$ & 94 & 48.0 \\
\hline $\mathrm{cN}+$ & 102 & 52.0 \\
\hline \multicolumn{3}{|l|}{ Tumor type } \\
\hline Ductal & 135 & 68.9 \\
\hline Lobular & 29 & 14.8 \\
\hline Other & 18 & 9.2 \\
\hline Unknown & 14 & 7.1 \\
\hline \multicolumn{3}{|l|}{ Menopausal status } \\
\hline Pre & 106 & 54.1 \\
\hline Peri & 9 & 4.6 \\
\hline Post & 79 & 40.3 \\
\hline Unknown & 2 & 1.0 \\
\hline \multicolumn{3}{|l|}{ Allocated treatment } \\
\hline Chemotherapy $+\mathrm{ZA}$ & 95 & 48.5 \\
\hline Chemotherapy only & 101 & 51.5 \\
\hline
\end{tabular}

$H R$ hormone receptor, $T N B C$ triple-negative breast cancer, ZA zoledronic acid

18.4\% in high CD8+ infiltrate vs. 5.2\% in low CD8+ infiltrate; $p=0.01)$. No statistical differences were observed in pCR rates when patients were divided based on the number of FoxP3+ (pCR: $12.5 \%$ in high FoxP3+ infiltrate vs. $9.0 \%$ in low FoxP3+ infiltrate; $p=0.49$ ) or CD68+ immune cells (pCR: $11.5 \%$ in high CD68+ infiltrate vs. $11.4 \%$ in low CD68+ infiltrate; $p=0.98$ ) (Fig. 2 and Online Resource 3).

Successful staining and scoring of HLA class 1 was achieved in $91 \%(n=178)$ and $87 \%(n=170)$ of biopsies, respectively. Based on these data, the HLA class 1 status of the tumor was determined in $91 \%(n=178)$. Tumors with loss or downregulation of HLA class 1 expression responded significantly worse to NAC than tumors with membranous HLA class 1 expression (pCR: $14.2 \%$ in tumors with HLA class 1 expression vs. $3.4 \%$ in tumors with HLA class 1 loss or downregulation; $p=0.03$ ) (Fig. 2 and Online Resource 3).
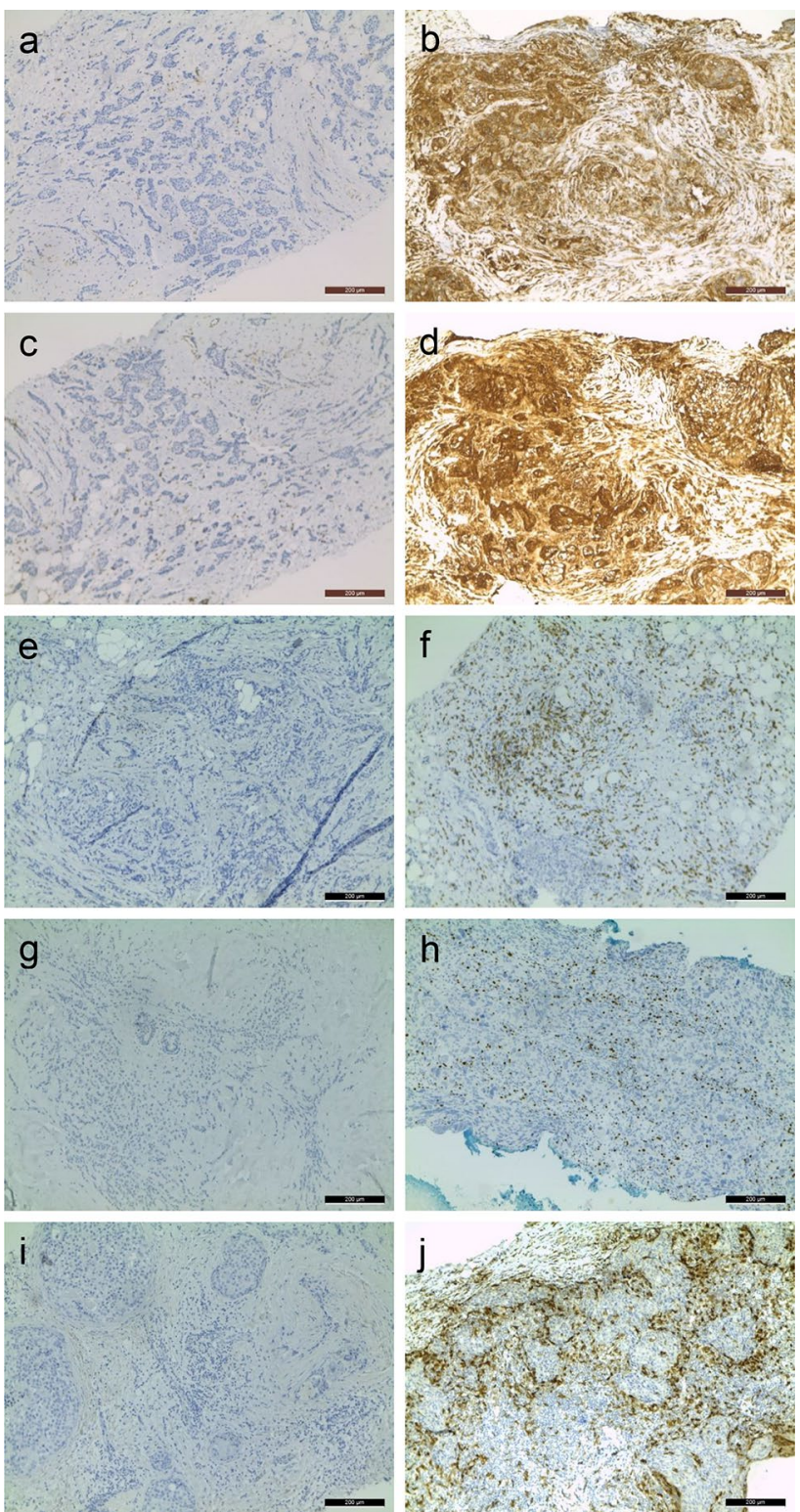

Fig. 1 Representative images of immunohistochemical stains for HLA class 1 (HCA2/HC10), CD8, FoxP3, and CD68. a HCA2negative tumor $(<5 \%$ of tumor cells staining positive for HCA2). b HCA2-positive tumor ( $\geq 5 \%$ of tumor cells staining positive for HCA2). c HC10-negative tumor. d HC10-positive tumor. e Tumor with low (below median) CD8+ infiltrate. $\mathbf{f}$ Tumor with high (above median) CD8+ infiltrate. $\mathbf{g}$ Tumor with low FoxP3 infiltrate. $\mathbf{h}$ Tumor with high FoxP3+ infiltrate. i Tumor with low CD68+ infiltrate. j Tumor with high CD68+ infiltrate. Images were taken at $\times 10$ magnification

The expression of HLA class 1 is a prerequisite for CD8+ CTLs to recognize tumor cells [36] and therefore the combination of these immune markers was evaluated further. In the presence of HLA class 1 expression, high CD8+ infiltrate was prognostic for $\mathrm{pCR}$ (pCR: $21.8 \%$ in high $\mathrm{CD} 8+$ infiltrate vs. $6.7 \%$ in low $\mathrm{CD} 8+$ infiltrate; $p=0.04$ ), whereas when 


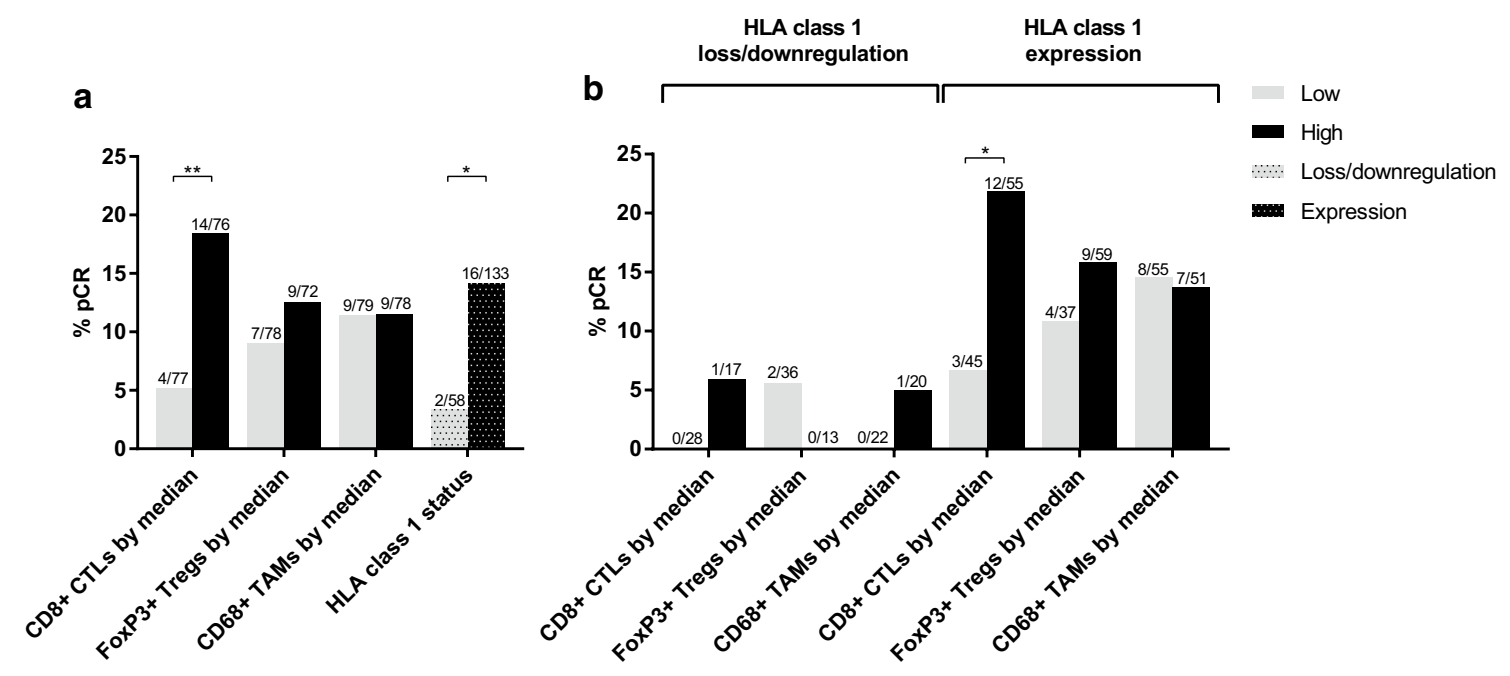

Fig. 2 Response (pCR) to NAC. a Stratified on immune markers b Based on immune markers, stratified on HLA class 1 status. Loss or downregulation of HLA class 1 is defined as $<5 \%$ of tumor cells staining positive for $\mathrm{HCA} 2$ and $\mathrm{HC} 10$ (loss), or either HCA2 or
HC10 (downregulation). Expression of HLA class 1 is defined as $\geq 5 \%$ of tumor cells staining positive for $\mathrm{HCA} 2$ and $\mathrm{HC} 10$. ${ }^{*} \mathrm{Chi}-$ square/Fisher's exact test $p<0.05$, **Chi-square test $p \leq 0.01$
HLA class 1 expression was lost or downregulated, there was no benefit of high CD8+ infiltrate (pCR: $5.9 \%$ in high CD8+ infiltrate vs. $0 \%$ in low CD8+ infiltrate; $p=0.38)$. No such association was found for FoxP3+ (in presence of HLA class 1 expression: $p=0.54$, loss or downregulation of HLA class 1 expression: $p=1.00$ ) or CD68+ infiltrate (in presence of HLA class 1 expression: $p=0.90$, loss or downregulation of HLA class 1 expression: $p=0.48$ ) (Fig. 2 and Online Resource 3 ). The balance between immune effector cells and immune suppressive cells is often used as a prognostic and predictive measure [22, 37]. When the group of patients was divided into four categories based on the combination of CD8+ and FoxP3+ infiltrate status (i.e., CD8 low/FoxP3 low, CD8 high/FoxP3 low, CD8 low/FoxP3 high, CD8 high/ FoxP3 high), no advantage for one group over the other was found ( $p=0.14$ ), neither when stratified on HLA class 1 status (in the presence of HLA class 1 expression: $p=0.43$, loss or downregulation of HLA class 1 expression: $p=0.59$ ).

The group of patients was then divided based on hormone receptor (HR) status, defined as negative when HER2, ER, and PR were negative (HR-/triple-negative breast cancer (TNBC)), and positive when ER and/or PR were positive $(\mathrm{HR}+)$. Patients with TNBC achieved more often a pCR (pCR: $26.7 \%$ (8 out of 30 ) in TNBC group vs. $8.2 \%$ (13 out of 159 ) in HR+ group; $p=0.008$ ). Furthermore, the TNBC group more often had HLA class I expression (86.2\% in TNBC group vs. $61.7 \%$ in $\mathrm{HR}+$ group; $p=0.01$ ) (Online Resource 1) and all seven patients with TNBC that achieved a pCR showed normal HLA class I expression, while six of these seven patients had strong CD8+ infiltrate. There were no differences in
CD8+ or CD68+ infiltrate between the TNBC and HR+ group ( $p=0.39$ and $p=0.80$, respectively), albeit that the numbers of FoxP3+ infiltrate were increased in the TNBC group $(80.8 \%$ FoxP3+ high infiltrate in TNBC group vs. $43.8 \%$ FoxP3 + high infiltrate in HR + group; $p=0.001)$ (Online Resource 1).

Allocated treatment-based analysis (chemotherapy plus or minus ZA), stratified on immune marker status, did not reveal any significant differences (Table 2) and neither did immune marker-based analysis, stratified on menopausal status (Online Resource 4). Immune marker-based analysis, stratified on HR status, showed that high CD8+ infiltrate and HLA expression are predictive for $\mathrm{pCR}$ in the HR+ group, but this is only a trend (pCR: $12.9 \%$ in high CD $8+$ infiltrate vs. $4.5 \%$ in low CD8+ infiltrate; $p=0.09$ and pCR: $10.0 \%$ in tumors with HLA class 1 expression vs. $3.7 \%$ in tumors with HLA class 1 loss or downregulation; $p=0.21$, respectively) (Online Resource 4).

Finally, multivariable analyses were performed to analyze the effect of each separate immune marker when corrected for hormonal status (positive or negative). This showed that the effect of CD8+ infiltrate on pCR was independent of hormonal status (OR 3.95, 95\% CI 1.21-12.90, $p=0.02$ ). Also, HLA class 1 expression influenced pCR rate irrespective of hormonal status (OR 3.78, 95\% CI 0.82-17.42, $p=0.09$ ), albeit this was a trend. No effect of FoxP3+ and CD68+ infiltrate was found (OR $0.89,95 \%$ CI $0.28-2.85, p=0.85$ and OR $0.97,95 \%$ CI $0.35-2.73, p=0.96$, respectively). 
Table 2 Allocated treatment-based analysis stratified on immune marker status

\begin{tabular}{|c|c|c|c|c|c|c|c|c|c|c|c|c|c|c|c|c|}
\hline \multicolumn{9}{|c|}{ CD8+ CTLs } & \multicolumn{8}{|c|}{ FoxP3+ Tregs } \\
\hline & \multicolumn{4}{|c|}{ Low $(<$ median $)$} & \multicolumn{4}{|c|}{ High ( $>$ median $)$} & \multicolumn{4}{|c|}{ Low $(<$ median $)$} & \multicolumn{4}{|c|}{$\operatorname{High}(>$ median $)$} \\
\hline & \multicolumn{2}{|c|}{ No pCR } & \multicolumn{2}{|c|}{$\mathrm{pCR}$} & \multicolumn{2}{|c|}{ No pCR } & \multicolumn{2}{|c|}{$\mathrm{pCR}$} & \multicolumn{2}{|c|}{ No pCR } & \multicolumn{2}{|c|}{$\mathrm{pCR}$} & \multicolumn{2}{|c|}{ No pCR } & \multicolumn{2}{|c|}{$\mathrm{pCR}$} \\
\hline & $\mathrm{N}$ & $\%$ & $\mathrm{~N}$ & $\%$ & $\mathrm{~N}$ & $\%$ & $\mathrm{~N}$ & $\%$ & $\mathrm{~N}$ & $\%$ & $\mathrm{~N}$ & $\%$ & $\mathrm{~N}$ & $\%$ & $\mathrm{~N}$ & $\%$ \\
\hline \multicolumn{17}{|c|}{ Allocated treatment } \\
\hline $\mathrm{ZA}+$ & 30 & $96.8 \%$ & 1 & $3.2 \%$ & 37 & $84.1 \%$ & 7 & $15.9 \%$ & 37 & $92.5 \%$ & 3 & $7.5 \%$ & 31 & $83.8 \%$ & 6 & $16.2 \%$ \\
\hline $\mathrm{ZA}-$ & 43 & $93.5 \%$ & 3 & $6.5 \%$ & 25 & $78.1 \%$ & 7 & $21.9 \%$ & 34 & $89.5 \%$ & 4 & $10.5 \%$ & 32 & $91.4 \%$ & 3 & $8.6 \%$ \\
\hline Total & 77 & & & & 76 & & & & 78 & & & & 72 & & & \\
\hline \multirow[t]{5}{*}{$p$ value } & 0.64 & & & & 0.51 & & & & 0.71 & & & & 0.48 & & & \\
\hline & \multicolumn{8}{|c|}{ CD68+ TAMs } & \multicolumn{8}{|c|}{ HLA class 1 status } \\
\hline & \multicolumn{4}{|c|}{ Low $(<$ median $)$} & \multicolumn{4}{|c|}{ High ( $>$ median) } & \multicolumn{4}{|c|}{ Loss + downregulation } & \multicolumn{4}{|c|}{ Expression } \\
\hline & \multicolumn{2}{|c|}{ No pCR } & \multicolumn{2}{|c|}{$\mathrm{pCR}$} & \multicolumn{2}{|c|}{ No pCR } & \multicolumn{2}{|c|}{$\mathrm{pCR}$} & \multicolumn{2}{|c|}{ No pCR } & \multicolumn{2}{|c|}{$\mathrm{pCR}$} & \multicolumn{2}{|c|}{ No pCR } & \multicolumn{2}{|c|}{$\mathrm{pCR}$} \\
\hline & $N$ & $\%$ & $N$ & $\%$ & $N$ & $\%$ & $N$ & $\%$ & $N$ & $\%$ & $N$ & $\%$ & $N$ & $\%$ & $N$ & $\%$ \\
\hline \multicolumn{17}{|c|}{ Allocated treatment } \\
\hline $\mathrm{ZA}+$ & 31 & $91.2 \%$ & 3 & $8.8 \%$ & 36 & $85.7 \%$ & 6 & $14.3 \%$ & 26 & $92.9 \%$ & 2 & $7.1 \%$ & 48 & $87.3 \%$ & 7 & $12.7 \%$ \\
\hline $\mathrm{ZA}-$ & 39 & $86.7 \%$ & 6 & $13.3 \%$ & 33 & $91.7 \%$ & 3 & $8.3 \%$ & 30 & $100.0 \%$ & 0 & $0.0 \%$ & 49 & $84.5 \%$ & 9 & $15.5 \%$ \\
\hline Total & 79 & & & & 78 & & & & 58 & & & & 113 & & & \\
\hline$p$ value & 0.73 & & & & 0.49 & & & & 0.23 & & & & 0.67 & & & \\
\hline
\end{tabular}

Loss or downregulation of HLA class 1 is defined as $<5 \%$ of tumor cells staining positive for HCA2 and HC10 (loss), or either HCA2 or HC10 (downregulation). Expression of HLA class 1 is defined as $\geq 5 \%$ of tumor cells staining positive for HCA2 and HC10. $p$ values represent Chi square of Fisher's exact tests. CTLs cytotoxic T-cells, TAMs tumor-associated macrophages, Tregs regulatory T-cells, ZA zoledronic acid

\section{Pre-treatment immune markers and associations with DFS}

Median follow-up was 4.6 years (cut-off date December 1, 2016). Upon stratification based on the median cell count per $\mathrm{mm}^{2}$ in the tumor area, a numerical but not significant trend towards a lower rate of DFS was observed in patients with high CD68+ infiltrate (hazard ratio 1.88, 95\% CI 0.83-4.26, $p=0.13)$. No statistical differences in DFS between patients with high or low CD8+ T-cell tumor infiltrate (hazard ratio $0.85,95 \%$ CI $0.39-1.88, p=0.70)$, high or low FoxP3+ T-cell tumor infiltrate (hazard ratio $1.55,95 \%$ CI 0.73-3.28, $p=0.25$ ), or expression or loss/downregulation of HLA class 1 of tumor cells were found (hazard ratio 1.38, 95\% CI $0.61-3.13, p=0.44)$. However, in the presence of HLA class 1 expression, patients with a tumor that was strongly infiltrated with CD8+ T-cells displayed a survival benefit, whereas in the absence or downregulation of HLA class 1 expression, this effect was lost ( $\mathrm{p}$ for interaction 0.01; hazard ratio $0.41,95 \%$ CI $0.15-1.10, p=0.08$ and hazard ratio 7.67 , 95\% CI 0.88-66.4, $p=0.07$, respectively) (Figs. 3, 4).

Similar analyses were performed for CD68+ and FoxP3+ infiltrate. CD68+ infiltrate was shown not to be prognostic in either subgroup (Fig. 4) and although FoxP3+ infiltrate was neither prognostic in patients with loss or downregulation of HLA class 1 expression, it tended to be inversely prognostic for DFS in patients with HLA class 1 expression (hazard ratio $2.22,95 \%$ CI $0.73-6.76, p=0.16$ ), fitting with its immune suppressive role (Figs. 4, 5).

When the group of patients was then divided into four categories based on the combination of CD8+ infiltrate and FoxP3+ infiltrate status as described above, no prognostic effect could be observed in the whole group (log-rank $p=0.65$ ). When stratified on HLA class 1 status, CD8+ high/FoxP3+ low infiltrate resulted in an improved DFS in the HLA class 1 expression group, although not significantly (log-rank $p=0.21$, p for interaction 0.69) (Fig. 6).

The ratio CD8+/CD68+ infiltrating immune cells was not prognostic in the whole group (hazard ratio $0.66,95 \% \mathrm{CI}$ $0.29-1.53, p=0.33)$, neither when stratified on HLA class 1 status ( $\mathrm{p}$ for interaction 0.86 ).

Additional subgroup analysis stratified on allocated treatment, HR status, and menopausal status did not reveal any significant differences except an inversely prognostic effect of CD68+ TAMs on DFS in premenopausal women (hazard ratio 2.85 , CI $1.03-7.88, p=0.04)$. However, the interaction test was not significant ( $p=0.56$ ) (Fig. 4). In HR+ patients, there was a trend for a better DFS $(p=0.19)$; however, HLA class 1 expression in combination with high CD8+ infiltrate was associated with an improved DFS, irrespective of the HR status (Online Resource 5). 
a HLA class 1 loss/downregulation

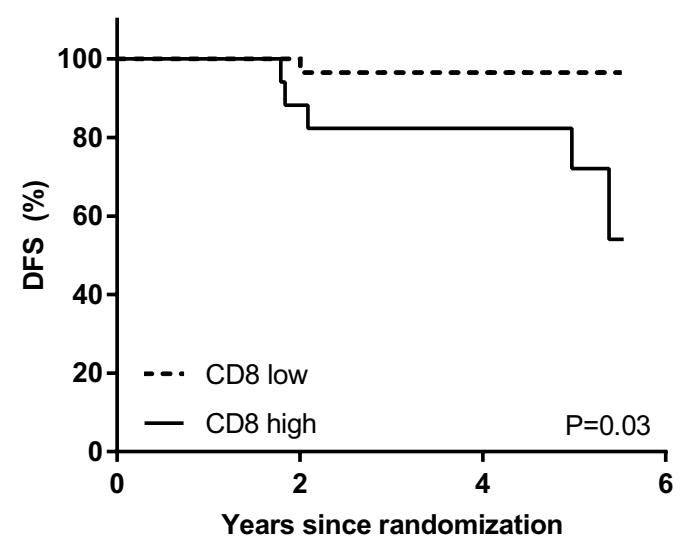

b HLA class 1 expression

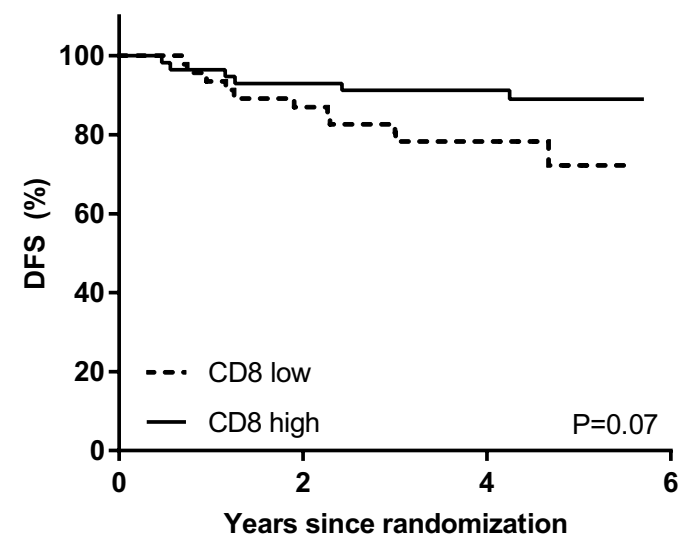

Fig. 3 Kaplan-Meier survival estimates based on CD8+ TILs, stratified on HLA class 1 status. a Effect of CD8+ tumor infiltrate on DFS in patients with HLA class 1 loss or downregulation. b Effect of
CD8+ tumor infiltrate on DFS in patients with HLA class 1 expression. $p$ values represent log-rank survival test

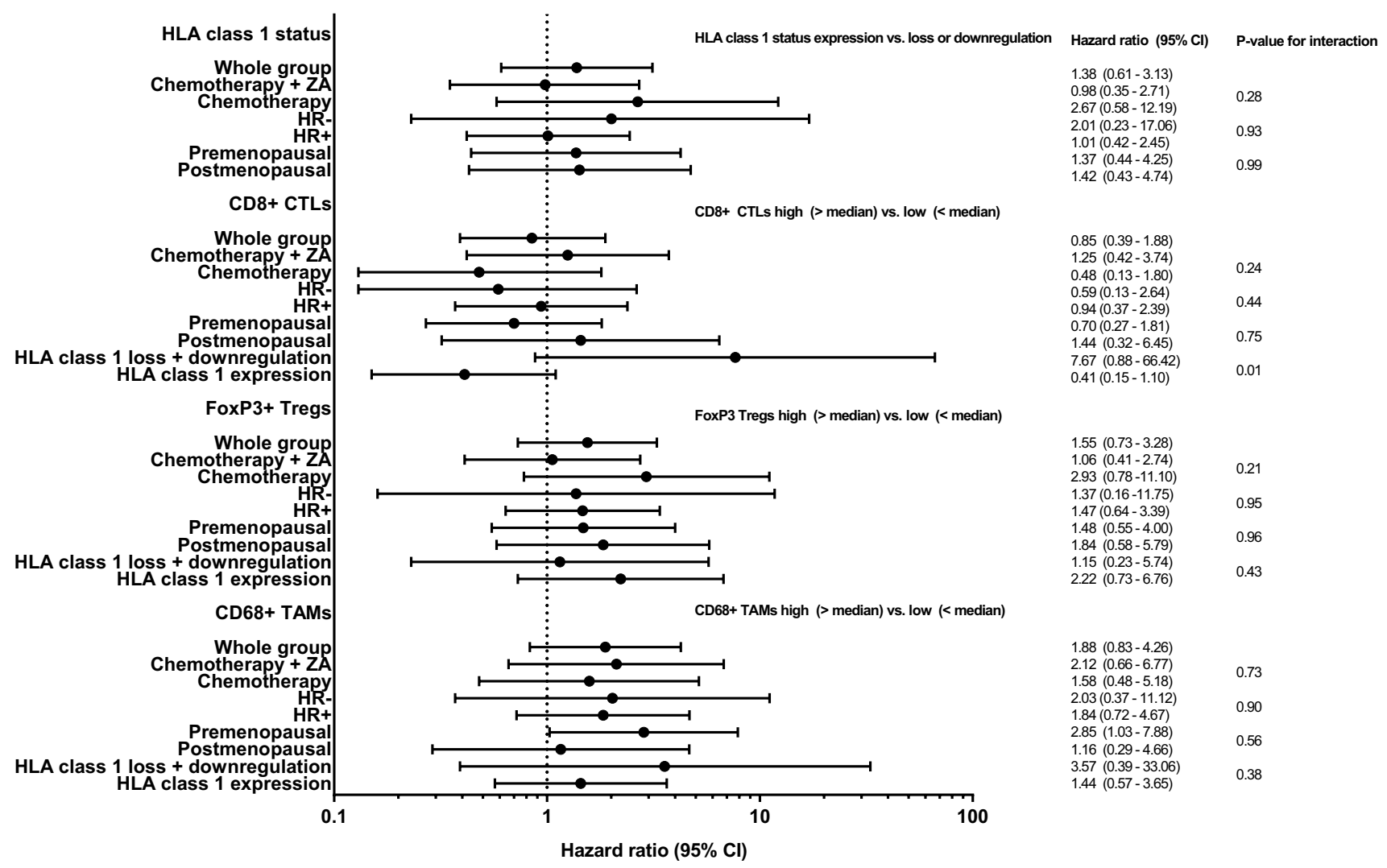

Fig. 4 Forest plot with immune marker-based (subgroup) analyses of DFS

\section{Discussion}

Our study shows that CD8+ TIL infiltrate and HLA class 1 status as individual markers are associated with higher
pCR rates after NAC. Neither of the immune markers was prognostic for DFS. However, when CD8+ TIL infiltrate was combined with HLA class 1 status, it became clear that higher pCR rates and a better DFS were detected in patients with a strongly CD8+ T-cell infiltrated tumor 
a

HLA class 1 loss/downregulation

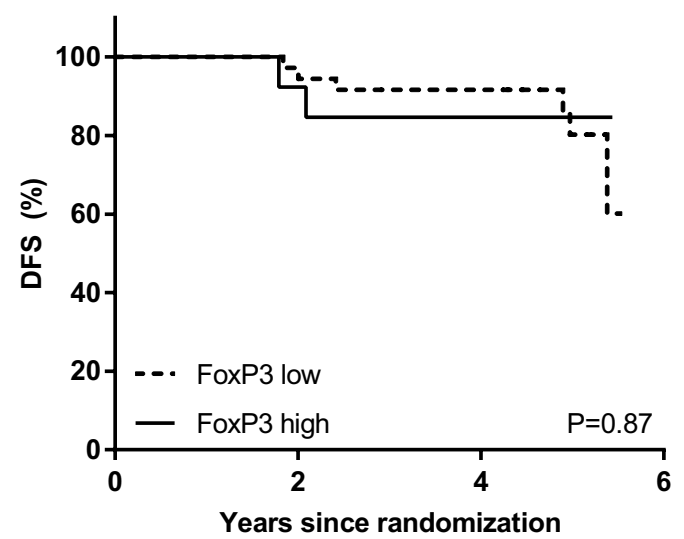

Fig. 5 Kaplan-Meier survival estimates based on FoxP3+ TILs, stratified on HLA class 1 status. a Effect of FoxP3+ tumor infiltrate on DFS in patients with HLA class 1 loss or downregulation. b Effect of b HLA class 1 expression

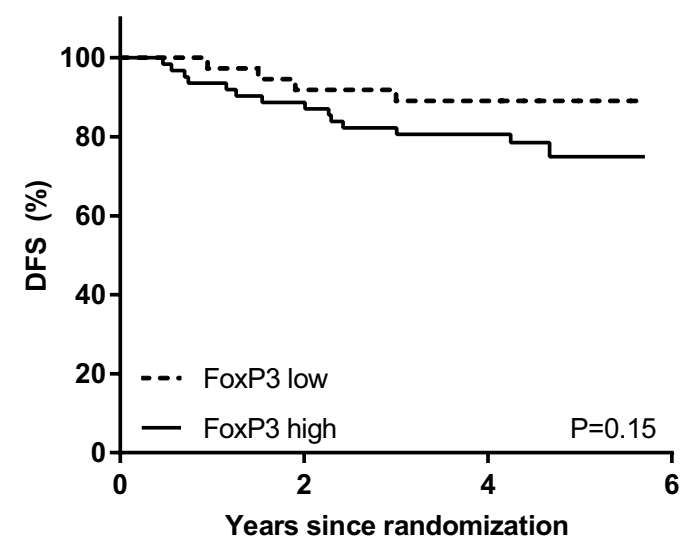

FoxP3+ tumor infiltrate on DFS in patients with HLA class 1 expression. $p$ values represent log-rank survival test

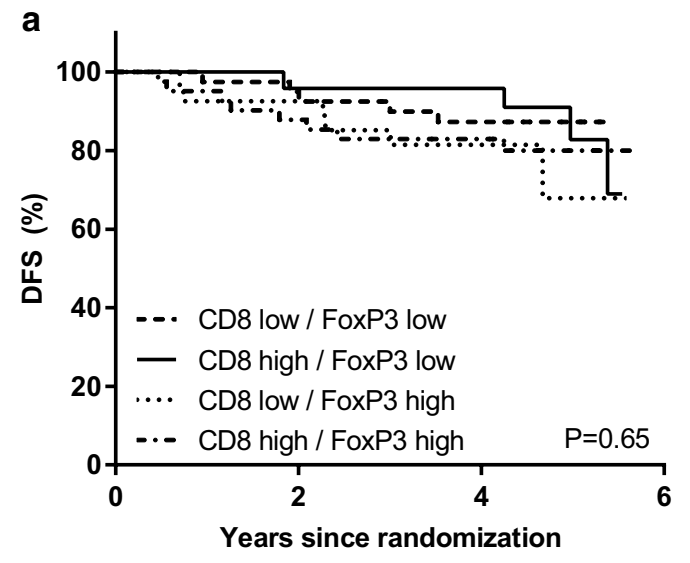

b HLA class 1 loss/downregulation

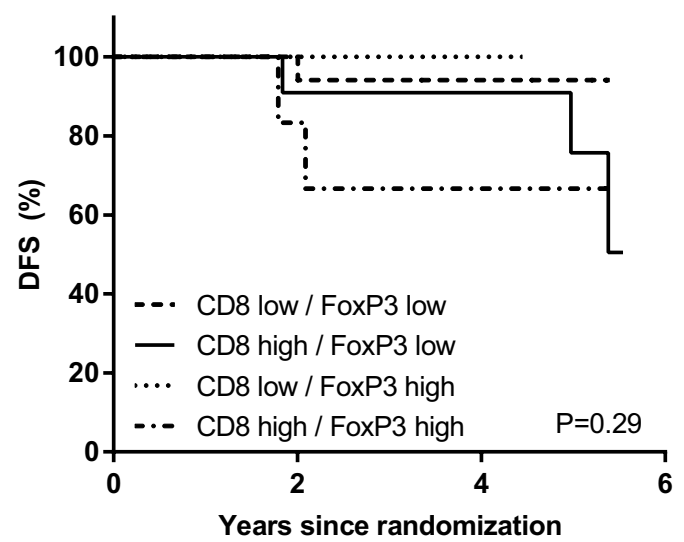

Fig. 6 Kaplan-Meier survival estimates based on the combination of CD8+ infiltrate and FoxP3+ infiltrate status. a Effect of the combination on DFS in the whole group. $\mathbf{b}$ Effect of the combination on DFS c HLA class 1 expression

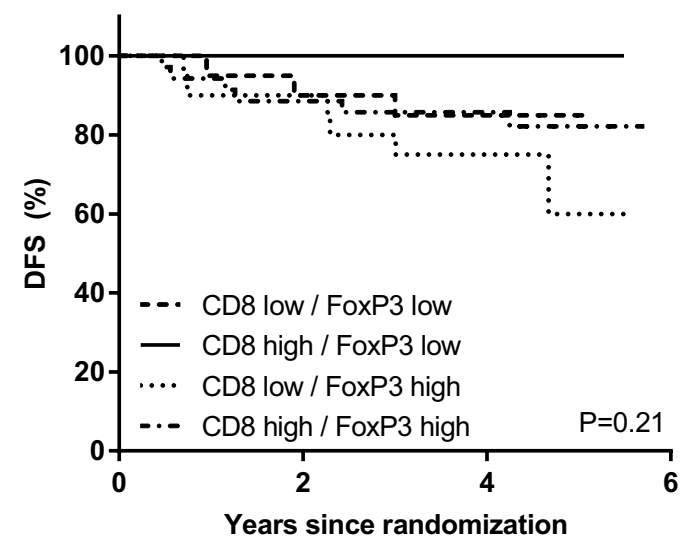

in patients with HLA class 1 loss or downregulation. $\mathbf{c}$ Effect of the combination on DFS in patients with HLA class 1 expression. $p$ values represent log-rank survival test 
which had retained a strong expression of HLA class 1 . To our knowledge, this is the first study investigating and defining the role of HLA class 1 expression in pCR after NAC.

Previous studies have concordantly proven TILs to be prognostic for outcome after NAC. However, the prognostic value of the different TIL subtypes is less clear. We found that high intratumoral CD8+ infiltrate, but not CD68+ and FoxP3+ infiltrate, correlates with a higher pCR. Furthermore, we observed a better pathological response of tumors strongly expressing HLA class 1 . We did not observe a direct relation between HLA class 1 status and survival as de Kruijf et al. did for chemotherapy treated patients. However, this might be explained by the fact that they studied a slightly different patient population (e.g., also patients with HER2positive tumors) and when chemotherapy was utilized, this was done in the adjuvant setting. In the NAC group, a beneficial effect on pCR and DFS was only observed when a strong infiltration of CD8+ T-cells coincided with a strong expression of HLA class 1 . This is explained by the fact that CD8+ CTLs are only capable of recognizing malignant cells if they express HLA class 1. Consequently, downregulation of HLA class 1 is commonly found in tumors as an immune escape mechanism [38].

TNBC is known to correlate with the presence of immune cells and a better response to chemotherapy than ER-positive breast cancer [7]. Indeed, the patient group with TNBC had a higher chance of achieving a pCR than those without TNBC. The small size of this group precludes strong statistically significant differences; however, the TNBC group more often had normal HLA class I expression and all TNBC patients achieving a pCR showed normal HLA class I expression, while all except one had stronger CD8+ T-cell infiltrate. There were no differences in CD8+ or CD68+ infiltration between patients with TNBC or without, albeit that the numbers of FoxP3+ cells were increased in TNBC. The latter, however, cannot explain why patients with TNBC would have relatively more pCRs. In the multivariable analyses with correction for hormonal status, the effect of CD8+ infiltrate on pCR was still significant and the effect of HLA class 1 status borderline significant.

Literature suggests that ZA is capable of eliciting an antitumor effect by modulating the immune system, specifically on gamma-delta T-cells [28] and on the recruitment and infiltration of TAMs [39-41]. Although we did not look into post-treatment immune changes in the tumor specimen, analysis of pre-treatment immune marker status did not reveal a relation between the number of TAMs and benefit from neoadjuvant $\mathrm{ZA}$ added to standard chemotherapy. Also, no treatment benefit of ZA for the other immune markers on pCR or DFS was found. Potentially, these pre-clinical data do not easily translate into the human setting. Another option is that because ZA as an adjunct to NAC did not improve pathological or clinical response to chemotherapy [27], no predictive immune biomarkers were found either. In addition, we did not study the effect of gamma-delta T-cells in the tumors.

Our study investigated TILs and TAMs that were located in the tumor cell nests. An international TILworking group recently postulated recommendations for evaluation of TILs on H\&E-stained slides [8-10]. As intraand peritumoral lymphocytes are hard to observe on $\mathrm{H} \& \mathrm{E}$ slides, the recommendations are based on TIL evaluation in the stromal compartment adjacent to the tumor in biopsies. However, with regard to the interaction between TILs and tumor cells, there is no biological rationale to use stromal TILs when intratumoral TILs can be visualized with immunohistochemistry $[9,10]$. For this reason, in our study, intratumoral TILs and TAMs were assessed.

Our study was limited regarding sample size as the study cohort was not prospectively powered for detecting statistical differences in pCR or DFS based on immune markers. Our current findings should be validated in an independent cohort with a similar population and treatment setting. Our current study was too small in sample size to do an adequate analysis of the most appropriate cut-off value. For this reason, the median of TIL and TAM cell counts was used for $\mathrm{CD} 8+/ \mathrm{FoxP} 3+$ and CD68+ status, respectively, similar to others $[42,43]$. One of the goals of a validation study should be to further investigate what an optimal cut-off value of cell counts may be for intratumoral CD8+ lymphocytes and the level of HLA class I downregulation that is still acceptable.

\section{Conclusions}

In summary, the new important finding for the clinic is that a strong baseline infiltration of early breast tumors with CD8+ T-cells is related to a higher pCR rate and a better DFS after NAC when the tumor cells have retained expression of HLA class 1 . Ultimately, it shows that the chemotherapy effect is also dependent on the immune system.

Acknowledgements We are greatly indebted to the participating patients and physicians, the local pathologists for providing tumor tissue, the Research Laboratory of the Department of Surgery of the Leiden University Medical Center for their help with the laboratory work, L. Verhoeff for clinical data collection, and B.E.W.M. van den Akker and N.F. de Miranda, PhD, for their help with the Vectra.

Funding This study was supported by grants from the Dutch Cancer Society (2010-4682), Amgen, Novartis, and Sanofi.

Data Availability The datasets used and/or analyzed during the current study are available from the corresponding author on reasonable request. 


\section{Compliance with ethical standards}

Conflict of interest The authors declare that they have no conflict of interest.

Ethical approval All procedures performed in studies involving human participants were in accordance with the ethical standards of the institutional and/or national research committee and with the 1964 Helsinki Declaration and its later amendments or comparable ethical standards. The NEOZOTAC trial was approved by the Medical Ethical Committee of the Leiden University Medical Center.

Informed consent Informed consent was obtained from all individual participants included in the study.

Open Access This article is distributed under the terms of the Creative Commons Attribution 4.0 International License (http://creativeco mmons.org/licenses/by/4.0/), which permits unrestricted use, distribution, and reproduction in any medium, provided you give appropriate credit to the original author(s) and the source, provide a link to the Creative Commons license, and indicate if changes were made.

\section{References}

1. Positive Results for Drug Combo in I-SPY 2 Trial (2014) Cancer Discov 4(2):OF2

2. Bonnefoi $\mathrm{H}$ et al (2014) Pathological complete response after neoadjuvant chemotherapy is an independent predictive factor irrespective of simplified breast cancer intrinsic subtypes: a landmark and two-step approach analyses from the EORTC 10994/ BIG 1-00 phase III trial. Ann Oncol 25(6):1128-1136

3. Cortazar P et al (2014) Pathological complete response and longterm clinical benefit in breast cancer: the $\mathrm{CTNeoBC}$ pooled analysis. Lancet 384(9938):164-172

4. Spring L et al (2016) Abstract 1439: pathological complete response after neoadjuvant chemotherapy predicts improved survival in all major subtypes of breast cancer: systematic review and meta-analyses of over 18,000 patients. Can Res 76(14 Supplement):1439-1439

5. Wang K et al (2016) Tumor-infiltrating lymphocytes in breast cancer predict the response to chemotherapy and survival outcome: a meta-analysis. Oncotarget 7(28):44288-44298

6. Carbognin L et al (2016) Predictive and prognostic role of tumorinfiltrating lymphocytes for early breast cancer according to disease subtypes: sensitivity analysis of randomized trials in adjuvant and neoadjuvant setting. Oncologist 21(3):283-291

7. Denkert $\mathrm{C}$ et al (2018) Tumour-infiltrating lymphocytes and prognosis in different subtypes of breast cancer: a pooled analysis of 3771 patients treated with neoadjuvant therapy. Lancet Oncol 19(1):40-50

8. Hendry $\mathrm{S}$ et al. (2017) Assessing tumor-infiltrating lymphocytes in solid tumors: a practical review for pathologists and proposal for a standardized method from the International Immunooncology Biomarkers Working Group: Part 1: assessing the host immune response, TILs in invasive breast carcinoma and ductal carcinoma in situ, metastatic tumor deposits and areas for further research. Adv Anat Pathol 24(5):235-251

9. Salgado R et al (2015) The evaluation of tumor-infiltrating lymphocytes (TILs) in breast cancer: recommendations by an International TILs Working Group 2014. Ann Oncol 26(2):259-271
10. Dieci MV et al (2018) Update on tumor-infiltrating lymphocytes (TILs) in breast cancer, including recommendations to assess TILs in residual disease after neoadjuvant therapy and in carcinoma in situ: a report of the International Immuno-Oncology Biomarker Working Group on Breast Cancer. Semin Cancer Biol 52(Pt 2):16-25

11. de Kruijf EM et al (2013) Tumor immune subtypes distinguish tumor subclasses with clinical implications in breast cancer patients. Breast Cancer Res Treat 142(2):355-364

12. de Kruijf EM et al (2010) The predictive value of HLA class I tumor cell expression and presence of intratumoral Tregs for chemotherapy in patients with early breast cancer. Clin Cancer Res 16(4):1272-1280

13. Rao N et al (2017) Significance of tumor-infiltrating lymphocytes and the expression of topoisomerase iialpha in the prediction of the clinical outcome of patients with triple-negative breast cancer after taxane-anthracycline-based neoadjuvant chemotherapy. Chemotherapy 62(4):246-255

14. Song IH et al (2017) Predictive Value of Tertiary Lymphoid Structures Assessed by High Endothelial Venule Counts in the Neoadjuvant Setting of Triple-Negative Breast Cancer. Cancer Res Treat 49(2):399-407

15. Miyashita M et al (2015) Prognostic significance of tumor-infiltrating CD8+ and FOXP3+ lymphocytes in residual tumors and alterations in these parameters after neoadjuvant chemotherapy in triple-negative breast cancer: a retrospective multicenter study. Breast Cancer Res 17:124

16. Mao $\mathrm{Y}$ et al (2014) The value of tumor infiltrating lymphocytes (TILs) for predicting response to neoadjuvant chemotherapy in breast cancer: a systematic review and meta-analysis. PLoS One 9(12):e115103

17. Demir L et al (2013) Predictive and prognostic factors in locally advanced breast cancer: effect of intratumoral FOXP3+ Tregs. Clin Exp Metastasis 30(8):1047-1062

18. Ladoire $S$ et al (2011) In situ immune response after neoadjuvant chemotherapy for breast cancer predicts survival. J Pathol 224(3):389-400

19. Liu F et al (2012) Peritumoral FOXP3(+) regulatory $\mathrm{T}$ cell is sensitive to chemotherapy while intratumoral FOXP3 $(+)$ regulatory $\mathrm{T}$ cell is prognostic predictor of breast cancer patients. Breast Cancer Res Treat 135(2):459-467

20. Zhao X et al (2017) Prognostic significance of tumor-associated macrophages in breast cancer: a meta-analysis of the literature. Oncotarget 8(18):30576-30586

21. Garcia-Martinez E et al (2014) Tumor-infiltrating immune cell profiles and their change after neoadjuvant chemotherapy predict response and prognosis of breast cancer. Breast Cancer Res 16(6):488

22. DeNardo DG et al (2011) Leukocyte complexity predicts breast cancer survival and functionally regulates response to chemotherapy. Cancer Discov 1(1):54-67

23. Zitvogel L, Tesniere A, Kroemer G (2006) Cancer despite immunosurveillance: immunoselection and immunosubversion. Nat Rev Immunol 6(10):715-727

24. Redondo M et al (2003) Major histocompatibility complex status in breast carcinogenesis and relationship to apoptosis. Hum Pathol 34(12):1283-1289

25. Madjd Z et al (2005) Total loss of MHC class I is an independent indicator of good prognosis in breast cancer. Int $\mathrm{J}$ Cancer 117(2):248-255

26. Gudmundsdottir I et al (2000) Altered expression of HLA class I antigens in breast cancer: association with prognosis. Int $\mathrm{J}$ Cancer 89(6):500-505

27. Charehbili A et al (2014) Addition of zoledronic acid to neoadjuvant chemotherapy does not enhance tumor response in patients 
with HER2-negative stage II/III breast cancer: the NEOZOTAC trial (BOOG 2010-01). Ann Oncol 25(5):998-1004

28. Hamilton E, Clay TM, Blackwell KL (2011) New perspectives on zoledronic acid in breast cancer: potential augmentation of anticancer immune response. Cancer Invest 29(8):533-541

29. McShane LM et al (2006) REporting recommendations for tumor MARKer prognostic studies (REMARK). Breast Cancer Res Treat 100(2):229-235

30. Sernee MF, Ploegh HL, Schust DJ (1998) Why certain antibodies cross-react with HLA-A and HLA-G: epitope mapping of two common MHC class I reagents. Mol Immunol 35(3):177-188

31. Seitz C et al (1998) The monoclonal antibody HCA2 recognises a broadly shared epitope on selected classical as well as several nonclassical HLA class I molecules. Mol Immunol 35(13):819-827

32. Perosa F et al (2003) Beta 2-microglobulin-free HLA class I heavy chain epitope mimicry by monoclonal antibody HC-10-specific peptide. J Immunol 171(4):1918-1926

33. Hutter $\mathrm{H}$ et al (1996) Expression of HLA class I molecules in human first trimester and term placenta trophoblast. Cell Tissue Res 286(3):439-447

34. Stam NJ, Spits H, Ploegh HL (1986) Monoclonal antibodies raised against denatured HLA-B locus heavy chains permit biochemical characterization of certain HLA-C locus products. J Immunol 137(7):2299-2306

35. Chew SF, Kanaan C, Tait BD (2007) HLA expression and cancer-14th IHIWS immunohistochemistry quality control exercise exchange results. Tissue Antigens 69(Suppl 1):248-251

36. Talebian Yazdi $M$ et al (2016) The positive prognostic effect of stromal CD8 + tumor-infiltrating T cells is restrained by the expression of HLA-E in non-small cell lung carcinoma. Oncotarget 7(3):3477-3488

37. Asano $Y$ et al (2016) Tumour-infiltrating CD8 to FOXP3 lymphocyte ratio in predicting treatment responses to neoadjuvant chemotherapy of aggressive breast cancer. Br J Surg 103(7):845-854

38. Cavallo F et al (2011) The immune hallmarks of cancer. Cancer Immunol Immunother 60(3):319-326

39. Rogers TL, Holen I (2011) Tumour macrophages as potential targets of bisphosphonates. J Transl Med 9:177

40. Rogers TL et al (2013) Macrophages as potential targets for zoledronic acid outside the skeleton-evidence from in vitro and in vivo models. Cell Oncol (Dordr) 36(6):505-514

41. Kroep JR et al (2016) Effects of neoadjuvant chemotherapy with or without zoledronic acid on pathological response: a meta-analysis of randomised trials. Eur J Cancer 54:57-63

42. Oda $\mathrm{N}$ et al (2012) Intratumoral regulatory $\mathrm{T}$ cells as an independent predictive factor for pathological complete response to neoadjuvant paclitaxel followed by 5-FU/epirubicin/cyclophosphamide in breast cancer patients. Breast Cancer Res Treat 136(1):107-116

43. Seo AN et al (2013) Tumour-infiltrating CD8 + lymphocytes as an independent predictive factor for pathological complete response to primary systemic therapy in breast cancer. Br J Cancer 109(10):2705-2713

Publisher's Note Springer Nature remains neutral with regard to jurisdictional claims in published maps and institutional affiliations. 